\title{
激光双焦点技术在高湍流度和稠密粒子 环境中的应用原理和实验方法
}

\author{
乐 㜊 E. D. Hirleman \\ (中国科学院力学研究所, 北京) (美国亚利桑那州立大学)
}

激光双焦点技术是近年内继多普勒技术之后发展起来的另一类测速技术 ${ }^{(3)}$. 由于相关和 非相关脉冲同时存在,通常又都忽略非相关脉冲的影响 ${ }^{[2]}$, 因而仅适用于低湍流度和低粒子密 度环境. 本文对整个脉冲信号序列进行了分析,提出了普适的实验方法. 此外,粒子通过焦点 的统计后效应作用是由湍流扩散决定的,脉冲信号序列的表达式直接与湍流特性有关 ${ }^{[3]}$.

\section{一、随机散射光脉冲序列的统计特性}

粒子群随气流穿越双焦点(每一焦点是一小体积元)发生散射光脉冲，相邻脉冲的间隔时 间定义为等待时间。显然,有两类近似独立的脉冲对构成总的信号序列，一是不同一粒子穿越 同一或不同一焦点产生的非相关脉冲对，另一类是同一粒子相继穿越双焦点产生的相关脉冲 对,它的等待时间表示了粒子在这一方向上的飞越时间.

1. 非相关穿越形成的复合泊松分布。按统计理论，若粒子随机分布于空间，平均浓度为 $c$, 在 $\boldsymbol{\imath}$ 时刻内, 有 $n$ 个随流体以速度 $U$ 移动的粒子通过焦点的概率是泊松分布

$$
W(t)=(\lambda t)^{n} \frac{e^{-\lambda t}}{n !},
$$

其中 $\lambda-U s c$, 表示平均到达率, $s$ 是焦点的迎风截面. 那么脉冲对的等待时间是 $t$ 的概率

$$
P_{t}^{1}=\lambda e^{-\lambda t} d t \text {. }
$$

当流体有湍流脉动, $\lambda$ 将随速度分布而变化为 $f(\lambda)^{[4]}$,

$$
P_{t}^{1}=\int_{-\infty}^{\infty} \lambda e^{-\lambda t} f(\lambda) d \lambda d t \text {. }
$$

在高斯速度分布时 $\quad P_{t}^{1}=\lambda_{0}\left(1-t \lambda_{0} \xi^{2}\right) \exp \left[-t \lambda_{0}+\frac{1}{2}\left(t \lambda_{0} \xi\right)^{2}\right] d t$ ，

$\lambda_{0}=U_{0} s c, \xi$ 是湍流强度.

2. 相关穿越的后效应作用. 粒子由湍流脉动产生随机位移, $t$ 时, 在下游焦点 $(B)$ 中的 某一点 $\left(\boldsymbol{r}_{2}\right)$ 存在一个粒子, 它在 $t=0$, 位于上游焦点 $(A)$ 中某一点 $\left(\boldsymbol{r}_{1}\right)$ 的概率 ${ }^{[5]}$ 是

$$
P_{a f}=\left(2 \pi Y^{2}\right)^{-3 / 2} \exp \left[-\frac{\left(r_{2}-\left(r_{1}+U_{t}\right)\right)^{2}}{2 Y^{2}}\right] \text {. }
$$

$Y^{2}$ 是扩散均方位移. 通过 $A$ 中任一点后会通过 $B$ 中任一点的概率就是

本文 1982 年 4 月 27 日收到。 


$$
P_{s}=\left(\frac{1}{V}\right) \int_{A} d r_{1} \int_{B} P_{a f} d r_{2} .
$$

$A, B$ 可近似看作直径为 $d$, 高为 $\Delta z$ 的圆柱, $V$ 为柱体积, 积分可得

$$
P_{s}=\left[\operatorname{erf} \alpha-\frac{1}{\alpha \sqrt{\pi}}\left(1-e^{-\alpha^{2}}\right)\right]\left[1-\left(I_{0} 2 \sqrt{\beta}+I_{1} 2 \sqrt{\beta}\right) e^{-2 \sqrt{\beta}}\right],
$$

其中 $\alpha=\frac{\Delta z}{2 \xi t U}, \beta=\frac{d}{4 \xi t U}$. 若速度分布为 $P_{g}$, 则粒子通过 $A$ 后必定通过 $B$ 的概率

$$
F_{c}^{1}=P_{s} \cdot P_{g} \text {. }
$$

高斯速度分布时

$$
P_{g}=\frac{\bar{t}}{\xi t^{2} \sqrt{2 \pi}} \exp \left[-\frac{1}{2 \xi^{2}}\left(\frac{\bar{t}}{t}-1\right)^{2}\right] d t,
$$

其中 $\bar{t}=L / U_{0}, L$ 是两个焦点之间的距离.

\section{二、应用原理和实验方法}

我们建立了一个新型的激光双焦点系统, 该系统的特点是由一个 2 "的方解石分束器同 时完成发射时的分束和接受时的合并作用, 由单个光电倍增管接受来自两个焦点的散射光脉 冲，经鉴别、转换，由多通道分析器得到序列等待时间的概率分布曲线，见图 1 .

按照一中的分析,此分布曲线的表达式为

$$
P_{t}=F_{c}+F_{U}-F_{c} \cdot F_{U}, F_{U}=P_{t}^{1}, F_{c}=\frac{1}{2} P_{s} \cdot P_{g} .
$$

将 (4), (7) 和 (9) 式代人, 图 2 是一组曲型的计算曲线.

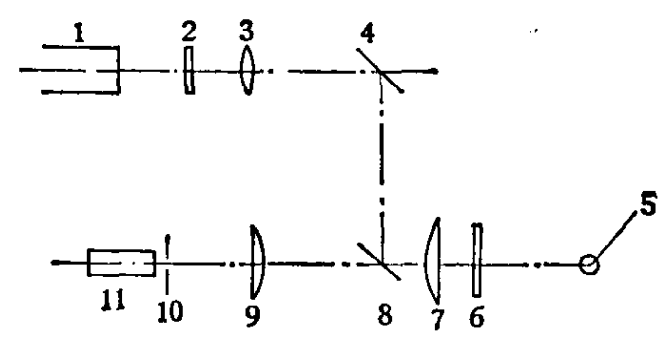

图 1 光学系统示意图

1一溦光器; $2-1 / 4$ 波片; $3,7,9$ ——透镜;

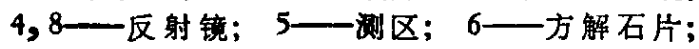
10——针孔; 11 一光电倍增管

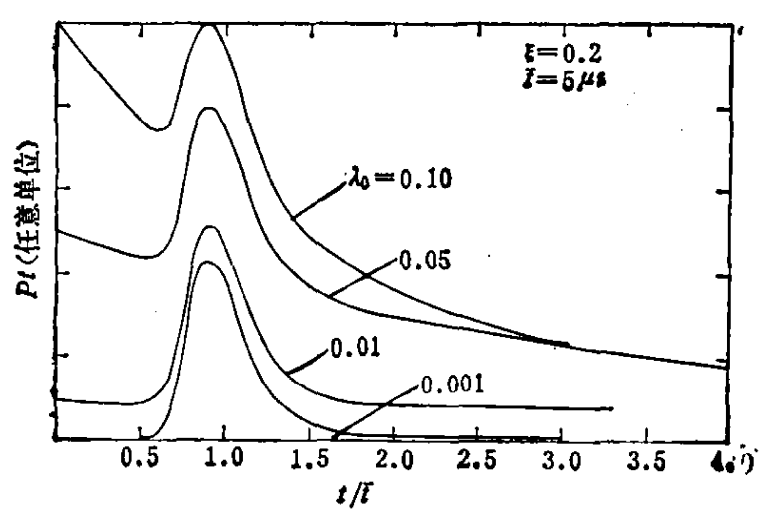

图 $2 P_{t}$ 的计算曲线

通常, 如果粒子浓度很低, 湍流度亦不大, 那么脉冲序列的特征可以近似地由高斯速度分 布来确定, 即忽略 $F_{U}$ ，而后效应作用作为一种误差, 虽可鉴别, 但无法排除 ${ }^{[6]}$. 湍流度上升, 粒 子浓度增加都使非相关部分增加. 单探测器系统也会导致非相关部分上升约四倍 ${ }^{[2]}$. 这时, 分 布曲线峰值对应时间与平均时间的差异上升，曲线变宽，这就是通常双焦点测速技术中，所得 速度和湍流度偏高的原因.

正确的实验方法是从实验分布曲线 (见图 3 中的照片)中分离出相关信息. 因为从数据采 集系统得到的分布曲线是分离和非正则化的, 故以 $i$ 表示 $i$ 通道的参数, $N_{\mathrm{J}}$ 为取样总数. 由 于 $P_{g}$ 的分布特点, 当 $t / \bar{t} \leqslant 0.2$ 时, $P_{g}\left(t_{i}\right) \ll P_{g}(\bar{t})$, 且 $F_{c, i} \ll F_{c, \max }$.

一个实验方法是在 $t / \bar{t} \leqslant 0.2$ 部分作曲线拟合, 然后在整个分布曲线中减去拟合得到的非 
相关部分,这只是一个数据处理问题.

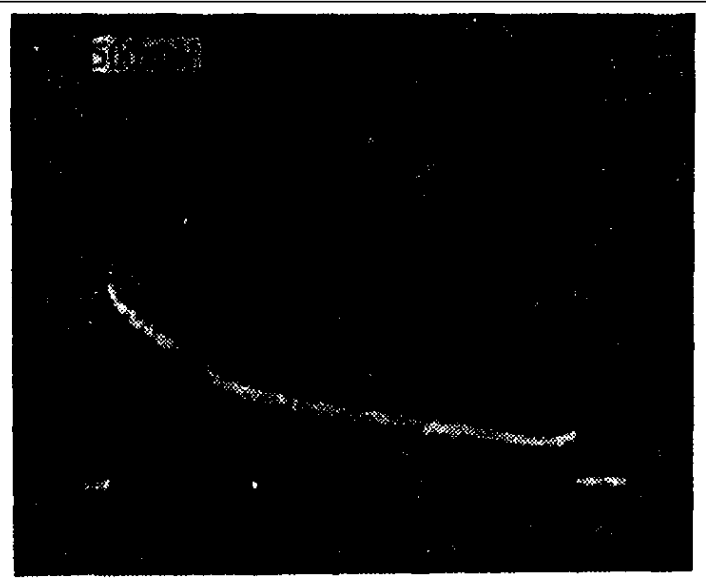

图 3 实验分布曲线

另一方法是分别在顺主气流和垂直主气流位 置置放双焦点，得到两条分布曲线

$$
\left\{\begin{array}{l}
N_{1, i}-N_{01}\left(F_{c, i}+F_{v, i}-F_{c i t} \cdot F_{v i i}\right), \\
N_{2, i}=N_{02} F_{v i i} .
\end{array}\right.
$$

用计算机控制采样时间达到 $N_{0 s} F_{v i}-N_{2, i}$, 使满 足 $t<0.2 \bar{z}, N_{1, i}-N_{2, i}$. 由 (11) 式得

$$
N_{0,1} F_{c, i}=\frac{N_{1, i}-N_{2 i d}}{1-\frac{N_{2 a i}}{N_{01}}} .
$$

最后，就是由统计加权平均求得平均速度和湍流 强度

$$
U=\Sigma t_{i} U_{i} F_{c i t} / \Sigma t_{i} F_{c i t},
$$

$$
\xi^{2}-\Sigma\left(\frac{1}{t_{i}} F_{c, i}\right)\left(\Sigma t_{i} F_{c, i}\right)-1 .
$$

图 4 和图 5 是用上述方法在一个高速自由射流中的测量结果．可以看出，若不考虑非相关背

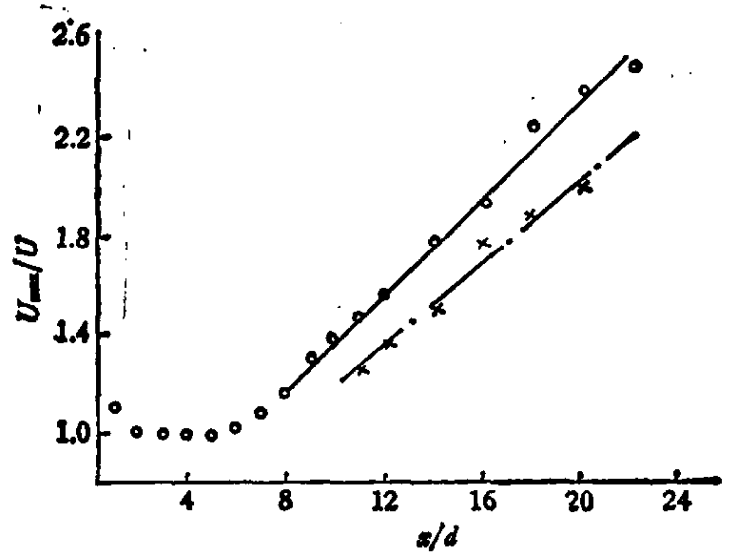

图 4 沿射流中心线的平均速度

○ 由式 (13) 确定的湖量值; + 由分布曲线值值 对应时间确定的住

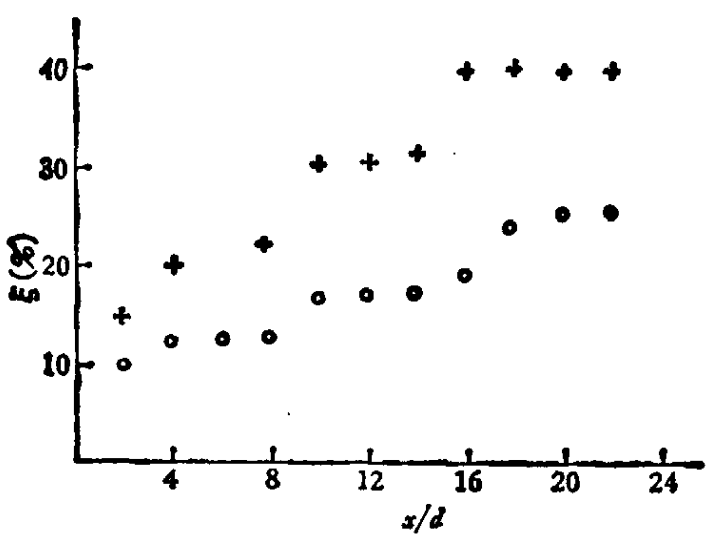

图 5 沿射流中心线的湍流强度

○ 由式 (14) 确定的阙五值; + 忽略非相关

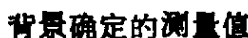

貫和植值时间与平均时间的差异将使平均速度和湍流强度的测量值偏高。

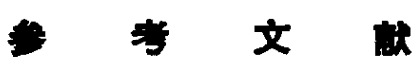

[1] Bchodl, R., A.G.A.B.D. Conf. Proc., No. 193, paper 21, 1976.

[2] Smart, A. E. \& Mayo, W. T., Proc. 4th Intermational Conf. on Photon Correlation Techniques in Fluid Mechanics, 1980.

[ 3 ] Erdmann, J. C. \& Gellert, R. I., Appl. Phys. Letters, 29(1976), 408.

[4]. Hirleman, E. D. \& Yao Yue, Research Report, Arizona State University, 1981.

[5] Chandrasekhar, S., Rov. Mod. Phys., 15(1943), 1.

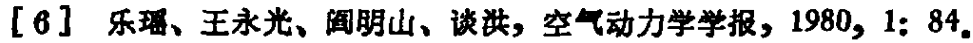

